\title{
Hot topic: Antilisterial activity by endolysin PlyP100 in fresh cheese
}

\author{
Maxwell L. Van Tassell, Luis A. Ibarra-Sánchez, Garrett P. Hoepker, and Michael J. Miller ${ }^{1}$ \\ Department of Food Science and Human Nutrition, University of Illinois, Urbana 61801
}

\begin{abstract}
Our objective was to assess the antimicrobial efficacy of a Listeria bacteriophage endolysin that may address limitations of current antilisterial processes for fresh cheeses. Listeria monocytogenes is highly problematic in the manufacture and processing of ready-to-eat foods due to its environmental persistence and its ability to grow under refrigerated storage. Special care must be taken to prevent listerial contamination during the production of fresh cheeses, as their delicate flavor and texture are incompatible with many of the antimicrobial processes and additives commonly used for other foods. Bacteriophage-derived cell wall hydrolytic enzymes, known as endolysins, comprise one possible intervention that may not suffer from the high strain specificity of their parent bacteriophages or the development of resistant strains. We recombinantly expressed endolysin PlyP100 and compared its lytic activity in vitro across several environmental parameters and target organisms, then incorporated it into a fresh cheese model challenged with a cocktail of $L$. monocytogenes. We show that PlyP100 demonstrates optimal activity under $\mathrm{pH}$ and salt concentrations consistent with a low-acid food matrix such as fresh cheese. Furthermore, we show that PlyP100 exhibits target specificity for gram-positive organisms with directly crosslinked peptidoglycan and displays considerable inhibitory activity against $L$. monocytogenes in fresh cheese for at least 4 wk under refrigerated storage. As PlyP100 demonstrates considerable promise for preventing the propagation of $L$. monocytogenes in fresh cheeses, this novel preservation method could help safeguard consumer health and the market expansion of an otherwise high-risk food with few other viable preservatives.
\end{abstract}

Key words: Listeria, cheese, endolysin, antimicrobial

\section{Hot Topic}

Listeria monocytogenes is a gram-positive foodborne pathogen responsible for considerable harm to public

Received September 12, 2016.

Accepted December 12, 2016.

${ }^{1}$ Corresponding author: mille216@illinois.edu health, particularly among the elderly, infants, pregnant women, and the immune-compromised. In these populations, infection can develop into deadly complications such as pneumonia, meningitis, septicemia, or uterine infections that can result in miscarriage or stillbirth. Dairy foods are linked to almost one-quarter of listeriosis cases, accounting for over $\$ 700$ million in healthcare costs annually in the United States alone (Batz et al., 2011). The majority of these cases are associated with soft cheeses, particularly fresh Hispanicstyle cheeses and surface-ripened cheeses. These favor the growth of $L$. monocytogenes because, unlike most cheeses, they have relatively low acid content, high moisture, and are often consumed without cooking. Preservation treatments involving high heat or pressure can negatively affect the texture or ripening properties of these cheeses and few chemical preservatives are as effective within the product $\mathrm{pH}$ range (Gould, 2000; Hnosko et al., 2012). Such limitations, along with the ability of $L$. monocytogenes to tolerate high salt content and grow under refrigeration, leave few effective control measures for these types of cheese. Due to these risk factors and the severity of listeriosis, US regulations completely prohibit the presence of L. monocytogenes at any level in ready-to-eat foods. This results in incalculable economic loss to the dairy industry regarding product testing, product loss, and recalls, as well as opportunity costs of not pursuing market expansion because of liability concerns.

Lytic bacteriophages (phages) have been pursued as a promising antimicrobial application for inclusion in food products to target specific pathogens. Considerable reduction in listerial load has even been achieved in fresh cheeses (Soni et al., 2012; Silva et al., 2014). Phages often feature narrow host spectra, however, with strain specificity limited to particular serovars. Broad-spectrum commercial phage preparations are now approved for use in the United States to inhibit $L$. monocytogenes in foods, but the development of strains resistant to phage infection raises concerns over their routine application as a preventative measure (Fister et al., 2016).

Bacteria can develop resistance to phages via several mechanisms. After successful infection, however, lysis is evoked by viral hydrolytic enzymes referred to as 
endolysins. As endolysins target conserved peptidoglycan (PG) structures in the cell wall, they exhibit less strain specificity than parental phages and are widely considered to be unlikely to promote the development of resistance in target organisms (Fischetti, 2005; Borysowski et al., 2006; Rodríguez-Rubio et al., 2016). Exogenous applications of endolysins have thereby been of considerable interest as alternative treatments for gram-positive pathogens, to overcome the limitations of antibiotics, phage therapies, and other antimicrobials.

However, only a few studies have characterized the antimicrobial activity of endolysins in food applications, primarily in milk over only short periods of time (Schmelcher and Loessner, 2016). We wished to assess the activity of an endolysin as an antimicrobial preservative in a fresh cheese for an elongated period under typical refrigerated storage, to reflect the nature of the problem posed by the gradual growth of $L$. monocytogenes in susceptible products. In a previous work, we developed a miniature fresh cheese model for assessing the efficacy of antimicrobials against Listeria (Van Tassell et al., 2015) in queso fresco. The objective for this study was to evaluate the potential for use of an endolysin as an antimicrobial in a fresh cheese matrix. To do so, we characterized the lytic activity of endolysin PlyP100, from L. monocytogenes phage P100, under various cheese-relevant conditions in vitro and incorporated PlyP100 into our model fresh cheese system.

The PlyP100 was prepared via recombinant expression. Using the sequence reported in the National Center for Biotechnology Information GenBank database (DQ004855.1), endolysin gene plyP100 was chemically synthesized and subcloned by Life Technologies (Thermo Fisher Scientific, Waltham, MA) into expression vector pRSET $B$ and transformed into competent Escherichia coli BL21 (DE3) using calcium chloride (Seidman et al., 2001). Overexpression of PlyP100 in the transformant culture was induced via isopropyl- $\beta$ D-thiogalactoside and purified using the QIAexpress Ni-NTA Fast Start Kit (Qiagen). Culture preparation, induction, expression conditions, and purification were carried out following the manufacturer's instructions, with a 20-h expression incubation at $25^{\circ} \mathrm{C}$. Purified PlyP100 was dialyzed into PBS $(\mathrm{KCl}, 200 \mathrm{mg} / \mathrm{L}$; $\mathrm{KH}_{2} \mathrm{PO}_{4}, 200 \mathrm{mg} / \mathrm{L} ; \mathrm{NaCl}, 8 \mathrm{~g} / \mathrm{L} ; \mathrm{Na}_{2} \mathrm{HPO}_{4}, 1.15 \mathrm{~g} / \mathrm{L}$, $\mathrm{pH}$ 7.2) via an Amicon Ultra-15 10K Centrifugal Filter Unit (Merck Millipore, Billerica, MA) and diluted with an equal volume of glycerol, followed by sterile filtration and storage at $-20^{\circ} \mathrm{C}$. Protein purity was confirmed via SDS-PAGE and quantified via the Quick Start Bradford Protein Assay (Bio-Rad, Hercules, CA) before use.

An ideal antilisterial should be able to target all possible strains of Listeria, but interfere with few other organisms. As an $N$-acetylmuramoyl-L-alanine amidase (Carlton et al., 2005), PlyP100 targets a PG linkage that is relatively conserved across all bacteria. To evaluate the breadth of the PlyP100 lytic spectrum, we compared its activity against a variety of Listeria and other gram-positive bacteria relevant to foods. Prior to preparation for each assay, bacterial strains (Table 1) were recovered from frozen glycerol stocks $\left(-80^{\circ} \mathrm{C}\right)$ by subculturing twice under conditions recommended for each strain by the Agricultural Research Service Culture Collection (NRRL; http://nrrl.ncaur.usda.gov) or American Type Culture Collection (https://www.atcc. org). All strains of Listeria were cultured aerobically with shaking $(250 \mathrm{rpm})$ in brain heart infusion broth (Becton, Dickinson and Co., Franklin Lakes, NJ) at $37^{\circ} \mathrm{C}$. Cultures were grown overnight, then washed with PBS and heat-killed $\left(121^{\circ} \mathrm{C}\right.$ for $\left.20 \mathrm{~min}\right)$ to maximize lytic activity and thereby sensitivity of the assay to detect susceptibility of each strain. Suspensions were then adjusted to a standardized optical density at $600 \mathrm{~nm}$ $\left(\mathbf{O D}_{600}\right)$ of 1.0 and combined 1:1 in microtiter plates with a final concentration of $2.5 \mu \mathrm{g} / \mathrm{mL}$ of PlyP100. The $\mathrm{OD}_{600}$ was measured immediately following mixture of the samples and after a 30-min incubation at $37^{\circ} \mathrm{C}$. Suspensions combined with buffer alone were used as untreated controls for each experimental treatment. Lytic activity was calculated as the percentage decrease in $\mathrm{OD}_{600}$ of treated samples, corrected for any decrease in $\mathrm{OD}_{600}$ of the controls, and reported as a percentage of the activity observed against L. monocytogenes 10403S.

The PlyP100 lysed each of the L. monocytogenes strains tested, across several serovars and of varied origin, as well as each strain of additional Listeria spp. tested (Table 1). This suggests that PlyP100 activity is not likely mediated by binding directly to the teichoic acid structures that vary among serovars and limit target range, as seen in several other listerial phage endolysins that have been characterized (Loessner et al., 2002; Schmelcher et al., 2010). Only a few of the other gram-positive organisms tested were partially sensitive to PlyP100; Bacillus subtilis, the target organism most closely related to Listeria, was as sensitive as the listerial strains, whereas the rest demonstrated much lower sensitivity. A very interesting finding of our specificity testing showed that, among all organisms tested, PlyP100 only affected strains of Schleifer and Kandler PG group A1 (Schleifer and Kandler, 1972), which are characterized by direct interpeptide cross-linkage between glycan chains. The presence of different crossbridging structure appears to prevent access of the PlyP100 catalytic site to the target $N$-acetylmuramoylL-alanine linkage. These observations are consistent 
VAN TASSELL ET AL.

Table 1. Lytic spectrum of PlyP100 against gram-positive bacterial strains used in this work

\begin{tabular}{|c|c|c|c|c|}
\hline Family, genus, and species & Strain identification ${ }^{1}$ & Sensitivity $( \pm \mathrm{SE})^{2}$ & $\mathrm{PG}^{3}$ & Serovar \\
\hline \multicolumn{5}{|l|}{ Listeriaceae } \\
\hline \multirow[t]{18}{*}{ Listeria monocytogenes } & $10403 \mathrm{~S}$ & $1.00 \pm 0.02$ & $\mathrm{~A} 1 \gamma$ & $1 / 2 \mathrm{a}$ \\
\hline & SLCC-5764 & $0.96 \pm 0.03$ & $\mathrm{~A} 1 \gamma$ & $1 / 2 \mathrm{a}$ \\
\hline & NRRL B-33419 & $0.97 \pm 0.04$ & $\mathrm{~A} 1 \gamma$ & $1 / 2 \mathrm{a}$ \\
\hline & NRRL B-33395 & $1.00 \pm 0.02$ & $\mathrm{~A} 1 \gamma$ & $1 / 2 \mathrm{a}$ \\
\hline & NRRL B-33391 & $0.91 \pm 0.04$ & $\mathrm{~A} 1 \gamma$ & $1 / 2 \mathrm{~b}$ \\
\hline & NRRL B-33424 & $0.89 \pm 0.02$ & $\mathrm{~A} 1 \gamma$ & $1 / 2 \mathrm{~b}$ \\
\hline & ATCC 7644 & $0.98 \pm 0.01$ & $\mathrm{~A} 1 \gamma$ & $1 / 2 \mathrm{c}$ \\
\hline & ATCC 19112 & $1.04 \pm 0.03$ & $\mathrm{~A} 1 \gamma$ & $1 / 2 \mathrm{c}$ \\
\hline & NRRL B-33393 & $1.01 \pm 0.03$ & $\mathrm{~A} 1 \gamma$ & $3 \mathrm{~b}$ \\
\hline & NRRL B-33226 & $0.97 \pm 0.02$ & $\mathrm{~A} 1 \gamma$ & $3 \mathrm{c}$ \\
\hline & NRRL B-33403 & $1.04 \pm 0.05$ & $\mathrm{~A} 1 \gamma$ & $4 a$ \\
\hline & ATCC 13932 & $0.95 \pm 0.01$ & $\mathrm{~A} 1 \gamma$ & $4 \mathrm{~b}$ \\
\hline & NRRL B-33420 & $0.97 \pm 0.01$ & $\mathrm{~A} 1 \gamma$ & $4 \mathrm{~b}$ \\
\hline & NRRL B-33513 & $0.90 \pm 0.05$ & $\mathrm{~A} 1 \gamma$ & $4 \mathrm{~b}$ \\
\hline & NRRL B-33104 & $1.00 \pm 0.01$ & $\mathrm{~A} 1 \gamma$ & $4 \mathrm{~b}$ \\
\hline & NRRL B-33231 & $0.89 \pm 0.05$ & $\mathrm{~A} 1 \gamma$ & $4 \mathrm{~b}$ \\
\hline & NRRL B-33116 & $1.00 \pm 0.04$ & $\mathrm{~A} 1 \gamma$ & $4 \mathrm{c}$ \\
\hline & NRRL B-33120 & $0.93 \pm 0.02$ & $\mathrm{~A} 1 \gamma$ & $4 \mathrm{~d}$ \\
\hline Listeria innocua & ATCC 33090 & $1.03 \pm 0.03$ & $\mathrm{~A} 1 \gamma$ & $4 \mathrm{e}$ \\
\hline Listeria innocua & NRRL B-33409 & $1.07 \pm 0.04$ & $\mathrm{~A} 1 \gamma$ & $6 \mathrm{a}$ \\
\hline Listeria ivanovii ssp. ivanovii & NRRL B-33017 & $0.96 \pm 0.04$ & $\mathrm{~A} 1 \gamma$ & $3 a$ \\
\hline Listeria welshimeri & NRRL B-33020 & $0.99 \pm 0.01$ & $\mathrm{~A} 1 \gamma$ & 5 \\
\hline Listeria seeligeri & NRRL B-33019 & $1.05 \pm 0.03$ & $\mathrm{~A} 1 \gamma$ & $6 \mathrm{~b}$ \\
\hline \multicolumn{5}{|l|}{ Lactobacillaceae } \\
\hline Lactobacillus delbrueckii ssp. bulgaricus & ATCC 11842 & $0.10 \pm 0.06$ & $\mathrm{~A} 4 \alpha$ & \\
\hline Lactobacillus casei & ATCC 393 & $0.15 \pm 0.02$ & $\mathrm{~A} 4 \alpha$ & \\
\hline Lactobacillus plantarum & ATCC 8014 & $0.44 \pm 0.11$ & $\mathrm{~A} 1 \gamma$ & \\
\hline Lactobacillus fermentum & NRRL B-1840 & $0.11 \pm 0.02$ & $\mathrm{~A} 4 \beta$ & \\
\hline Lactobacillus brevis & NRRL B-4527 & $0.01 \pm 0.13$ & $\mathrm{~A} 4 \alpha$ & \\
\hline Lactobacillus rhamnosus & ATCC 53103 & $0.16 \pm 0.11$ & $\mathrm{~A} 4 \alpha$ & \\
\hline Pediococcus acidilactici & NRRL B-14958 & $0.05 \pm 0.03$ & $\mathrm{~A} 4 \alpha$ & \\
\hline \multicolumn{5}{|l|}{ Carnobacteriaceae } \\
\hline Carnobacterium divergens & NRRL B-14830 & $0.08 \pm 0.04$ & $\mathrm{~A} 1 \gamma$ & \\
\hline \multicolumn{5}{|l|}{ Enterococcaceae } \\
\hline Enterococcus faecium & ATCC 6057 & $0.00 \pm 0.05$ & $\mathrm{~A} 4 \alpha$ & \\
\hline \multicolumn{5}{|l|}{ Aerococcaceae } \\
\hline Aerococcus viridans & ATCC 11563 & $0.48 \pm 0.05$ & $\mathrm{~A} 1 \alpha$ & \\
\hline \multicolumn{5}{|l|}{ Streptococcaceae } \\
\hline Lactococcus lactis ssp. cremoris & ATCC 19257 & $0.03 \pm 0.12$ & $\mathrm{~A} 4 \alpha$ & \\
\hline Lactococcus lactis ssp. lactis & ATCC 19435 & $0.03 \pm 0.04$ & $\mathrm{~A} 4 \alpha$ & \\
\hline \multicolumn{5}{|l|}{ Leuconostocaceae } \\
\hline Leuconostoc mesenteroides & NRRL B-1118 & $0.10 \pm 0.01$ & $\mathrm{~A} 3 \alpha$ & \\
\hline Weissella confusa & NRRL B-1064 & $0.09 \pm 0.05$ & $\mathrm{~A} 3 \alpha$ & \\
\hline \multicolumn{5}{|l|}{ Bacillaceae } \\
\hline Bacillus subtilis & NRRL NRS-744 & $0.94 \pm 0.16$ & $\mathrm{~A} 1 \gamma$ & \\
\hline \multicolumn{5}{|l|}{ Clostridiaceae } \\
\hline Clostridium difficile & ATCC 70057 & $0.53 \pm 0.07$ & $\mathrm{~A} 1 \gamma$ & \\
\hline Clostridium perfringens & ATCC 13124 & $0.21 \pm 0.08$ & $\mathrm{~A} 3 \gamma$ & \\
\hline \multicolumn{5}{|l|}{ Staphylococcaceae } \\
\hline Staphylococcus aureus & NRRL B-767 & $0.09 \pm 0.03$ & $\mathrm{~A} 3 \alpha$ & \\
\hline \multicolumn{5}{|l|}{ Bifidobacteriaceae } \\
\hline Bifidobacterium bifidum & NRRL B-41410 & $0.06 \pm 0.17$ & $\mathrm{~A} 4 \beta$ & \\
\hline \multicolumn{5}{|l|}{ Brevibacteriaceae } \\
\hline Brevibacterium linens & NRRL B-4210 & $0.49 \pm 0.06$ & $\mathrm{~A} 1 \gamma$ & \\
\hline
\end{tabular}

${ }^{1}$ SLCC $=$ special Listeria culture collection; NRRL = Agricultural Research Service Culture Collection (http:// nrrl.ncaur.usda.gov); ATCC = American Type Culture Collection (https://www.atcc.org).

${ }^{2}$ Sensitivity to PlyP100, as measured by turbidity reduction of heat-killed cell suspensions incubated with the enzyme $(2.5 \mu \mathrm{g} / \mathrm{mL})$ for $30 \mathrm{~min}$, relative to L. monocytogenes $10403 \mathrm{~S}$.

${ }^{3}$ Peptidoglycan chemotype subgroup of the strain's cell wall, according to Schleifer and Kandler (1972) and Schleifer (2009). 
with the specificity observed among homologous enzymes, including the listerial phage endolysins LysZ5 (Zhang et al., 2012) and Ply511 (Loessner et al., 1995).

As environmental conditions are important factors in enzymatic activity, we measured the effect of 3 key variables ( $\mathrm{pH}$, salt concentration, and temperature) on the enzymatic activity of PlyP100. As PlyP100 exhibited comparable activity against the PG of all Listeria strains tested, lytic assays were carried out as described above against Listeria innocua American Type Culture Collection 33090. Cultures and buffers were prepared as described by Linden et al. (2015) to vary salt concentrations from 0 to $500 \mathrm{mM} \mathrm{NaCl}$ and $\mathrm{pH}$ of 3 to 11 . To assess the effect of temperature on lytic activity, cell suspensions and buffers were pre-incubated and incubated between measurements at a given temperature of 4 to $50^{\circ} \mathrm{C}$.

Under optimal conditions, the concentrations of PlyP100 tested could generally elicit a 70 to $80 \%$ reduction in turbidity in $30 \mathrm{~min}$. The activity of PlyP100 was optimal at near-neutral $\mathrm{pH}$ (Figure 1A), with little activity observed below $\mathrm{pH} 6$ or above $\mathrm{pH}$ 10. Minimal activity at $\mathrm{pH} 5$ or below suggests that PlyP100 may not be a viable option in acidified foods. However, most unripened fresh cheeses maintain a $\mathrm{pH}$ around 6 (Guo et al., 2011; Caro et al., 2014), which is not acidic enough for the effective use of many other preservatives at low concentration (Davidson et al., 2005). The PlyP100 activity was also high within the range of salt content seen in many fresh cheeses (Guo et al., 2011), about 1 to $2 \%$ or up to about $350 \mathrm{mM} \mathrm{NaCl}$, with optimal activity observed at approximately 100 to $150 \mathrm{mM}$ $\mathrm{NaCl}$ in phosphate buffer (Figure 1B). Furthermore, although PlyP100 activity declined below $37^{\circ} \mathrm{C}$ (Figure $1 \mathrm{C}$ ), it remained high up to $50^{\circ} \mathrm{C}$, so enzymatic activity should be maintained throughout the temperatures of cheese manufacture.
Observations of this environmental dependency suggested innumerable other factors may influence the antimicrobial potential of PlyP100. Uncertain whether enzymatic activity could be maintained in a more complex milieu than buffered cell suspensions, and under refrigerated storage, we sought practical in situ validation of the antimicrobial application in our target environment: queso fresco. Therefore we incorporated PlyP100 into fresh cheeses inoculated with a cocktail of food- and human-isolates of foodborne outbreakassociated L. monocytogenes strains. Miniaturized laboratory fresh cheeses were prepared as described previously (Van Tassell et al., 2015), inoculated with a cocktail of L. monocytogenes: NRRL strains B-33104, B33419, B-33420, B-33424, and B-33513. The PlyP100 was added to the drained curd, before final pressing, at a final concentration of $750 \mu \mathrm{g}$ per g of cheese. Phosphate-buffered saline was incorporated into untreated cheeses. Cheeses with and without PlyP100 were stored at $4^{\circ} \mathrm{C}$ for up to $28 \mathrm{~d}$ and enumerated on PALCAM Listeria-Selective agar (EMD Millipore, Billerica, MA) for listerial survival.

Listeria monocytogenes grew well in untreated queso fresco under refrigerated storage at $4^{\circ} \mathrm{C}$, increasing from 5 to $8 \mathrm{log} \mathrm{cfu} / \mathrm{g}$ in about 2 wk (Figure 2). The PlyP100 appeared to act largely inhibitory in queso fresco, reducing cell viability by only about one half-log $\mathrm{cfu} / \mathrm{g}$ from the starting inoculum, but preventing further growth for over 3 wk. In soy milk, endolysin LysZ5 was shown to elicit a several-log colony-forming unit reduction of listerial viability within hours (Zhang et al., 2012). Perhaps a similarly extensive and immediate reduction in viability of the inocula was not observed in the presence of PlyP100 because enzymatic mobility was more limited within a cheese matrix and may have obstructed physical access of the enzyme to some target cells. Furthermore, some component of milk may
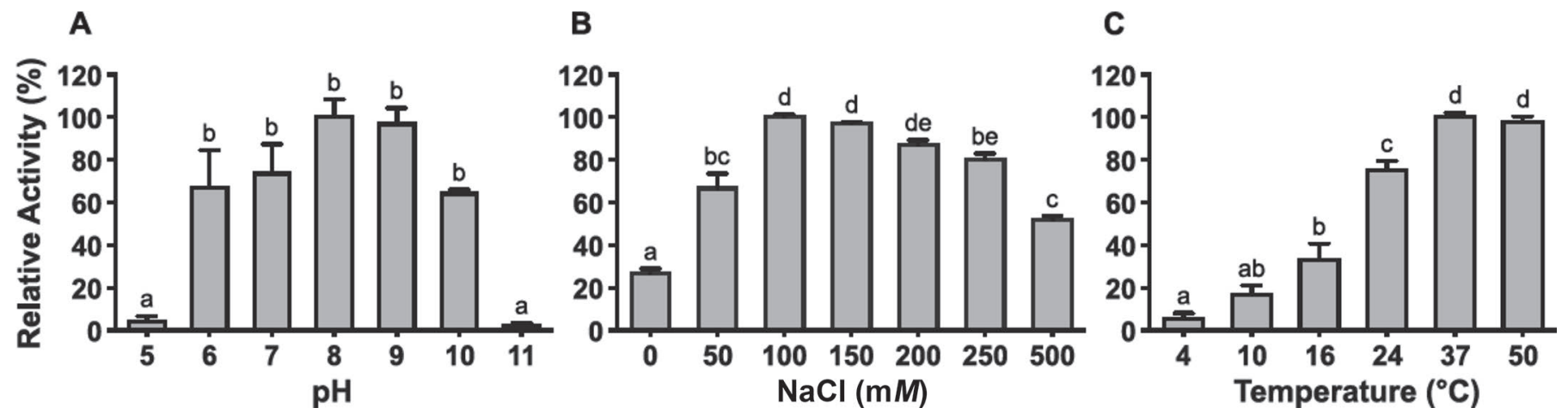

Figure 1. Optimal environmental conditions for lytic activity of PlyP100. The optimal (A) pH, (B) salt concentration, and (C) temperature for the lysis of Listeria innocua American Type Culture Collection 33090 were characterized via turbidity reduction assay over 30 min using 10 $\mu \mathrm{g} / \mathrm{mL}$ of PlyP100. The optimal activity for each variable was set as $100 \%$. Bars indicate the mean \pm standard error of triplicate independent experiments. Values sharing a letter within each panel are not significantly different $(P<0.05)$. 


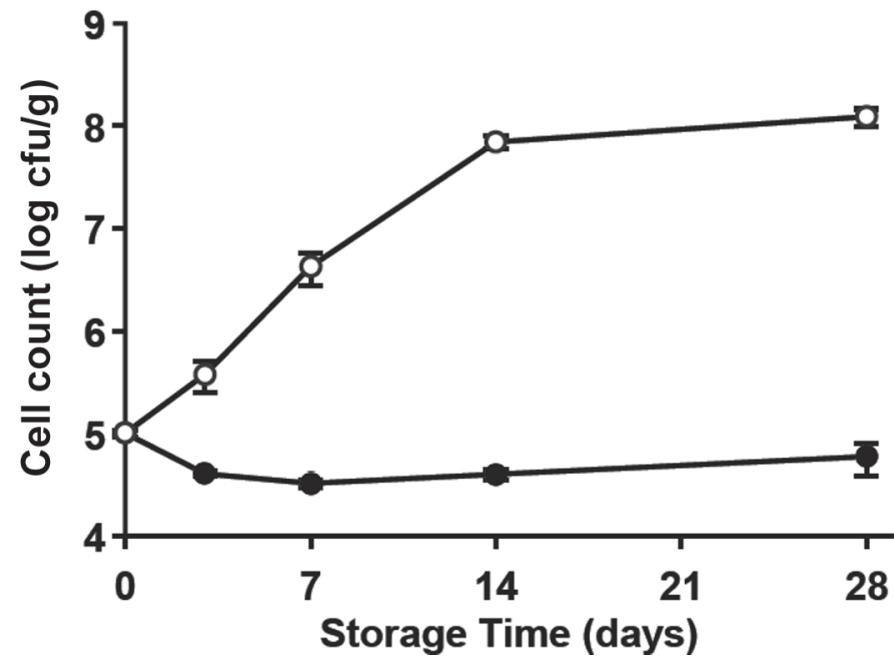

Figure 2. Antimicrobial activity of PlyP100 against Listeria monocytogenes in a fresh cheese model over $4 \mathrm{wk}$ of storage at $4^{\circ} \mathrm{C}$. The enzyme was added to cheeses by weight, with final concentrations of 0 (open circle) and 750 (closed circle) $\mu \mathrm{g} / \mathrm{g}$ of PlyP100. Values are means \pm standard error of triplicate independent experiments.

interact with the enzymes directly to limit bactericidal activity relative to activity in less complex suspensions. It is important to note, however, that antimicrobial testing in buffer or microbiological medium cannot necessarily be extrapolated to a food system, or even between solid and fluid food systems. For example, Soni et al. (2010) showed that a 4-fold increase in concentration of lauric arginate was required in milk for antimicrobial activity comparable in tryptic soy broth, with still greater concentrations required in cheese as well. However, to our knowledge, we have demonstrated the first use of an endolysin for preservation of a dairy product over the course of its shelf life. Treatment with PlyP100 prevented a $3 \mathrm{log} \mathrm{cfu} / \mathrm{g}$ growth of L. monocytogenes relative to the untreated control over $4 \mathrm{wk}$, with an approximately $0.5 \mathrm{log} \mathrm{cfu} / \mathrm{g}$ reduction relative to the inocula.

Overall, PlyP100 appears largely compatible with dairy products in terms of environmental tolerance and microbial specificity; not only will it likely inhibit the majority of, if not all, listerial strains, but it also seems unlikely to negatively affect the desirable bacteria native to most dairy fermentations based on our observations of specificity. An endolysin of such appropriate spectrum may also be advantageous for the prevention of dairy spoilage, targeting the equivalent PG structure in the Bacillaceae that comprise most common gram-positive isolates of spoiled dairy (Trmčić et al., 2015). Furthermore, PlyP100 is effective at inhibiting the growth of Listeria in laboratory fresh cheeses and warrants follow-up for further application in dairy manufacturing.

As such, endolysins exhibit considerable potential for addressing listerial contamination in dairy products. They may be effective when incorporated into other cheese products, such as smear-ripened or mold-ripened cheeses that also more readily harbor Listeria due to de-acidification that takes place during surface ripening. Endolysins could be paired with other, traditionally less effective antimicrobial preservatives to screen for synergistic combinatorial interventions. A small-scale cheese model could also be used for a targeted screening or bioengineering of endolysins to alter their structural characteristics, such as size, charge, or conformation, and thereby their catalytic efficiency, environmental tolerance, or diffusivity through food matrices (Van Tassell et al., 2016).

Considerable work remains before successful commercial application of recombinant endolysins can be seen in foods, including matters of scale, cost-efficiency, and regulatory approval. Further study is needed to assess enzyme stability, dose-response, and timing of their introduction in contaminated cheeses to optimize application criteria and begin addressing some of these concerns. Moreover, safety for human consumption will need to be validated before establishing guidelines for endolysin use in foods, which do not currently exist. However, this work has contributed to our understanding of the effects of endolysins in fresh cheeses and provided the necessary proof-of-concept for their implementation through extended storage.

\section{ACKNOWLEDGMENTS}

We thank the Miller laboratory group for thoughtful discussion of the manuscript and the USDA Agricultural Research Service for contribution of the strains from the NRRL Culture Collection. This study was supported by the USDA Cooperative State Research, Education and Extension Service (Washington, DC), Hatch project \#ILLU-698-339 to MJM, Universidad Autónoma de Querétaro-University of Illinois Small Research Grants Program, CONACYT PhD scholarship for LAIS, and the University of Illinois Graduate College Dissertation Completion Fellowship for MLVT.

\section{REFERENCES}

Batz, M. B., S. Hoffman, and J. G. Morris Jr. 2011. Ranking the Risks: The 10 Pathogen-Food Combinations With the Greatest Burden on Public Health. Emerging Pathogens Institute, University of Florida, Gainesville.

Borysowski, J., B. Weber-Dabrowska, and A. Górski. 2006. Bacteriophage endolysins as a novel class of antibacterial agents. Exp. Biol. Med. (Maywood) 231:366-377. 
Carlton, R. M., W. H. Noordman, B. Biswas, E. D. de Meester, and M. J. Loessner. 2005. Bacteriophage P100 for control of Listeria monocytogenes in foods: Genome sequence, bioinformatic analyses, oral toxicity study, and application. Regul. Toxicol. Pharmacol. 43:301-312.

Caro, I., S. Soto, L. Fuentes, N. Gutiérrez-Méndez, B. García-Islas, K. E. Monroy-Gayosso, and J. Mateo. 2014. Compositional, functional and sensory characteristics of selected Mexican cheeses. Food Nutr. Sci. 05:366-375.

Davidson, P. M., J. N. Sofos, and A. L. Branen, ed. 2005. Antimicrobials in Food. 3rd ed. Taylor \& Francis, Boca Raton, FL.

Fischetti, V. A. 2005. Bacteriophage lytic enzymes: Novel anti-infectives. Trends Microbiol. 13:491-496.

Fister, S., S. Fuchs, B. Stessl, D. Schoder, M. Wagner, and P. Rossmanith. 2016. Screening and characterisation of bacteriophage P100 insensitive Listeria monocytogenes isolates in Austrian dairy plants. Food Contr. 59:108-117.

Gould, G. W. 2000. Preservation: Past, present and future. Br. Med. Bull. 56:84-96.

Guo, L., D. L. Van Hekken, P. M. Tomasula, J. Shieh, and M. H. Tunick. 2011. Effect of salt on the chemical, functional, and rheological properties of Queso Fresco during storage. Int. Dairy J. $21: 352-357$.

Hnosko, J., M. F. San-Martin Gonzalez, and S. Clark. 2012. Highpressure processing inactivates Listeria innocua yet compromises Queso Fresco crumbling properties. J. Dairy Sci. 95:4851-4862.

Linden, S. B., H. Zhang, R. D. Heselpoth, Y. Shen, M. Schmelcher, F Eichenseher, and D. C. Nelson. 2015. Biochemical and biophysical characterization of PlyGRCS, a bacteriophage endolysin active against methicillin-resistant Staphylococcus aureus. Appl. Microbiol. Biotechnol. 99:741-752.

Loessner, M. J., K. Kramer, F. Ebel, and S. Scherer. 2002. C-terminal domains of Listeria monocytogenes bacteriophage murein hydrolases determine specific recognition and high-affinity binding to bacterial cell wall carbohydrates. Mol. Microbiol. 44:335-349.

Loessner, M. J., G. Wendlinger, and S. Scherer. 1995. Heterogeneous endolysins in Listeria monocytogenes bacteriophages: A new class of enzymes and evidence for conserved holin genes within the siphoviral lysis cassettes. Mol. Microbiol. 16:1231-1241.

Rodríguez-Rubio, L., D. Gutiérrez, D. M. Donovan, B. Martínez, A. Rodríguez, and P. García. 2016. Phage lytic proteins: Biotechnological applications beyond clinical antimicrobials. Crit. Rev. Biotechnol. 36:542-552.

Schleifer, K.-H. 2009. Phylum XIII. Firmicutes Gibbons and Murray 1978, 5 (Firmacutes [sic] Gibbons and Murray 1978, 5). Pages
19-1317 in Systematic Bacteriology. W. B. Whitman, ed. Springer New York, New York, NY.

Schleifer, K. H., and O. Kandler. 1972. Peptidoglycan types of bacterial cell walls and their taxonomic implications. Bacteriol. Rev. $36: 407-477$.

Schmelcher, M., and M. J. Loessner. 2016. Bacteriophage endolysins: Applications for food safety. Curr. Opin. Biotechnol. 37:76-87.

Schmelcher, M., T. Shabarova, M. R. Eugster, F. Eichenseher, V. S. Tchang, M. Banz, and M. J. Loessner. 2010. Rapid multiplex detection and differentiation of Listeria cells by use of fluorescent phage endolysin cell wall binding domains. Appl. Environ. Microbiol. 76:5745-5756.

Seidman, C. E., K. Struhl, J. Sheen, and T. Jessen. 2001. Introduction of plasmid DNA into cells. Pages 1.8.1-1.8.10 in Current Protocols in Molecular Biology. F. M. Ausubel, R. Brent, R. E. Kingston, D. D. Moore, J. G. Seidman, J. A. Smith, and K. Struhl, ed. John Wiley \& Sons Inc., Hoboken, NJ.

Silva, E. N. G., A. C. L. Figueiredo, F. A. Miranda, and R. C. de Castro Almeida. 2014. Control of Listeria monocytogenes growth in soft cheeses by bacteriophage P100. Braz. J. Microbiol. 45:11-16.

Soni, K. A., M. Desai, A. Oladunjoye, F. Skrobot, and R. Nannapaneni. 2012. Reduction of Listeria monocytogenes in queso fresco cheese by a combination of listericidal and listeriostatic GRAS antimicrobials. Int. J. Food Microbiol. 155:82-88.

Soni, K. A., R. Nannapaneni, M. W. Schilling, and V. Jackson. 2010. Bactericidal activity of lauric arginate in milk and Queso Fresco cheese against Listeria monocytogenes cold growth. J. Dairy Sci. 93:4518-4525.

Trmčić, A., N. H. Martin, K. J. Boor, and M. Wiedmann. 2015. A standard bacterial isolate set for research on contemporary dairy spoilage. J. Dairy Sci. 98:5806-5817.

Van Tassell, M. L., M. A. Daum, J.-S. Kim, and M. J. Miller. 2016. Creative lysins: Listeria and the engineering of antimicrobial enzymes. Curr. Opin. Biotechnol. 37:88-96.

Van Tassell, M. L., L. A. Ibarra-Sánchez, S. R. Takhar, S. L. AmayaLlano, and M. J. Miller. 2015. Use of a miniature laboratory fresh cheese model for investigating antimicrobial activities. J. Dairy Sci. 98:8515-8524.

Zhang, H., H. Bao, C. Billington, J. A. Hudson, and R. Wang. 2012. Isolation and lytic activity of the Listeria bacteriophage endolysin LysZ5 against Listeria monocytogenes in soya milk. Food Microbiol. 31:133-136. 